\title{
miR-20a-directed regulation of BID is associated with the TRAIL sensitivity in colorectal cancer
}

\author{
GUANLI HUANG $^{1}$, XIANGJIAN $\mathrm{CHEN}^{1}$, YEFENG CAI $^{2}$, XIAOBO WANG $^{2}$ and $\mathrm{CHUNGEN} \mathrm{XING}^{1}$ \\ ${ }^{1}$ Department of General Surgery, The Second Affiliated Hospital of Soochow University, Suzhou, Jiangsu 215004; \\ ${ }^{2}$ Department of Surgical Oncology, The First Affiliated Hospital of Wenzhou \\ Medical University, Wenzhou, Zhejiang 325000, P.R. China
}

Received June 1, 2016; Accepted October 31, 2016

DOI: $10.3892 /$ or.2016.5278

\begin{abstract}
MicroRNAs (miRNAs) are small, non-coding RNAs that play important roles in cancer processes. Although miR-20a has been reported to be altered in a range of cancers, the role of miR-20a in colorectal cancer is not fully characterized, and the relationship between miR-20a dysregulation and the tumor necrosis factor-related apoptosis-inducing ligand (TRAIL) sensitivity is not defined. In the present study, we demonstrated significant upregulation of miR-20a in the serum of colorectal cancer patients, tumor tissues and cell lines by quantitative RT-PCR analysis. Furthermore, we found that the TRAIL-induced apoptosis was associated with the expression level of miR-20a in colorectal cancer. The knockdown of miR-20a by inhibitors increased the antitumor effect of TRAIL via caspase- 8 dependent pathway. BID, which is a pro-apoptotic member of the Bcl-2 family, was found to be directly regulated by miR-20a in SW480 cells. The knockdown of miR-20a inhibited the translocation of tBID to the mitochondria, which induced the mitochondrial pathway of apoptosis. Notably, we found that the knockdown of miR-20a also reversed the resistance of TRAIL in established TRAIL-resistant SW480 cells by tBID-mitochondria pathway. Therefore, our results suggest that miR-20a acts as a tumor promoter in colorectal cancer, and the understanding of the miR-20a might be a potential therapeutic target for colorectal cancer.
\end{abstract}

\section{Introduction}

Colorectal cancer is the third most common cancer and the leading cause of cancer-related mortality around the world (1). The high mortality of colorectal cancer is due to the high rate of synchronous liver metastases which is found in newly

Correspondence to: Dr Chungen Xing, Department of General Surgery, The Second Affiliated Hospital of Soochow University, No. 1055 Sanxian Road, Suzhou, Jiangsu 215004, P.R. China E-mail: xingcg@qq.com

Key words: colorectal cancer, miR-20a, TRAIL, BID, caspase-8 diagnosed colorectal cancer patients. Therefore, colorectal cancer is one of the diseases seriously threating health $(2,3)$. For patients with advanced colorectal cancer, tumor resection is not feasible and chemotherapy and biotherapy have become the only strategies for treatment $(4,5)$. Unfortunately, however, these treatments are mostly ineffective because of the development of chemoresistance in cancer patients (6).

TNF-related apoptosis-inducing ligand (TRAIL) is a member of the TNF superfamily, which can induce extrinsic apoptosis depending on the activation of caspase-8 (7). TRAIL has been considered as a potential therapeutic agent in cancer treatment due to its capability to induce apoptosis selectively in tumor cells, but not in normal cells (8). Therefore, unlike TNF- $\alpha$ or chemotherapeutics, TRAIL shows little toxicity when administered in vivo. However, a significant proportion of cancer cells exhibit low sensitivity to TRAIL-induced apoptosis, which precludes its use in many cases $(9,10)$. It is urgent to find a strategy to increase the sensitivity of tumor cells to TRAIL.

MicroRNAs (miRNAs) are endogenous, small noncoding RNAs consisting of approximately 21-25 nucleotides (nt) (11). They are involved in post-transcriptional control of approximately $60 \%$ of the human genes by binding to the 3'-untranslated region (3'-UTR) of target mRNAs. Therefore, miRNAs participate in a wide array of biological processes including cell proliferation, development, differentiation, apoptosis and others $(12,13)$. Aberrant miRNA expression has been frequently observed in various human tumors, which was indicated to be involved in cancer development and progression (14). In the present study, we demonstrated that miR-20a was dysregulated in colorectal cancer and associated with the TRAIL sensitivity. Therefore, this study presents significant insight into the role of miR-20a, which shows potential for developing miRNA-related colorectal cancer therapy.

\section{Materials and methods}

Clinical specimens and cell lines. Thirty pairs of colorectal cancer tissues and the adjacent non-tumor tissues were obtained from the patients who underwent tumor resection in The First Affiliated Hospital of Wenzhou Medical University from June 2013 to September 2015. Both tumor 
and non-cancerous samples were confirmed histologically. In addition, 80 serum samples from 40 colorectal cancer patients and 40 healthy controls were collected from the same hospital before the treatment. All samples were collected with patients' informed consent, and this project was approved by the Ethics Committee of The First Affiliated Hospital of Wenzhou Medical University. FHC which is a normal colorectal epithelial cell line (15) and the colorectal cancer cell lines SW480, SW948, NCI-H508 and HT-29 were originated from the American Type Culture Collection (ATCC; Manassas, VA, USA). SW480, SW948, NCI-H508 and HT-29 cells were cultured in Dulbecco's modified Eagle's medium (DMEM) supplemented with $10 \%$ fetal bovine serum (FBS; Gibco- Invitrogen), $100 \mathrm{U} /$ $\mathrm{ml}$ penicillin and $100 \mu \mathrm{g} / \mathrm{ml}$ streptomycin. FHC cells were cultured as previously described (15). All cells were cultured at $37^{\circ} \mathrm{C}$ with $5 \% \mathrm{CO}_{2}$. TRAIL resistant SW480 (R-SW480) was established by continuous exposure to TRAIL. Briefly, SW480 cells were treated with TRAIL for $48 \mathrm{~h}$ and then the cells were moved to the TRAIL-free medium for another $72 \mathrm{~h}$ after drug treatment. This process was repeated three times before the cells were exposed to the next higher dose of TRAIL. The initiating concentration of TRAIL was $1 \mathrm{ng} /$ $\mathrm{ml}$, and the concentration was increased by $0.5 \mathrm{ng} / \mathrm{ml}$ up to a final concentration of $5 \mathrm{ng} / \mathrm{ml}$. The R-SW480 cells were cultured in medium with TRAIL, but before the experiments were performed, R-SW480 cells were moved to the TRAIL-free medium for 2 weeks.

Quantitative real-time polymerase chain reaction ( $q R T-P C R)$. RNA was extracted from frozen tissues, serum and colorectal cancer cell lines using the TRIzol reagent (Invitrogen, Carlsbad, CA, USA) according to the manufacturer's instructions. The reverse transcription of miR-20a was performed by stem-loop RT primer using the PrimeScript RT reagent kit (Takara, Dalian, China), and quantified by qPCR using the SYBR Premix Ex Taq (Takara) according to the manufacturer's instructions. The primers were synthesized as follows: miR-20a for RT primer: 5'-CTCAACTGGTGTCGTGGAGT CGGCAATTCAGTTGAGATTTCACG-3'. QPCR forward primer: 5'-ACACTCCAGCTGGGGATGGACGTGATATT CG-3' and reversed primer: 5'-TGGTGTCGTGGAGTCG-3'. U6 snRNA was used as the internal control. The relative expression of miR-20a was determined using the $2^{-\Delta \Delta C \mathrm{~T}}$ analysis method (16).

Transfection. BID siRNA, miR-20a mimics, miR-20a inhibitor (anti-miR-20a) and the negative control (miR-NC) were synthesized by Guangzhou RiboBio, Co., Ltd. (Guangzhou, China). The SW480 cells were transfected with $50 \mathrm{pmol} / \mathrm{ml}$ BID SiRNA (5'-GAAGACAUCAUCCGGAAUAUU-3'), $50 \mathrm{pmol} / \mathrm{ml} \mathrm{miR}-20$ a mimics (5'-GAUGGACGUGAUAUUC GUGAAAU-3'), $50 \mathrm{pmol} / \mathrm{ml}$ 2'-Omethyl modified antimiR-20a (5'-AUUUCACGAAUAUCACGUCCAUC-3'), $50 \mathrm{pmol} / \mathrm{ml} \mathrm{miR-NC} \mathrm{(5'-GUCGAGAUUGGAUGAUAAUC}$ AUG-3') using Lipofectamine 2000 (Invitrogen) according to the manufacturer's guidance.

Luciferase reporter assay. The 3'-UTR fragment of BID containing the seed region was amplified and inserted downstream of the luciferase reporter gene in the luciferase reporter pMIR-Report-Vector (Life Technologies, Carlsbad, CA, USA). The mutant 3'-UTR of BID (GCACUUU to GCAGAUU) was amplified by wild-type reporter containing BID $3^{\prime}$-UTR as the template, and the mutant plasmid was created by Site-Directed Mutagenesis kit (Takara). For luciferase reporter assays, the SW480 cells were co-transfected with RNAs and luciferase reporters. Following transfection for $48 \mathrm{~h}$, the cells were collected and lysed. Luciferase activity was then measured by using Dual-luciferase assay (Promega, Madison, WI, USA) according to the manufacturer's instructions. Firefly luciferase activity was normalized to the Renilla luciferase activity, and the ratio of firefly/Renilla was presented.

Cell viability assay. For cell viability assay, $24 \mathrm{~h}$ post-transfection of RNAs, the cells were reseeded in 96-well plates at a density of $2 \times 10^{3}$ cells/well. Subsequently, after the cells were treated with TRAIL for $48 \mathrm{~h}$, they were stained with $20 \mu \mathrm{l}$ 3-(4,5-dimethylthiazol-2-yl)-2,5-diphenyltetrazolium bromide (MTT) $(5 \mathrm{mg} / \mathrm{ml}$; Sigma-Aldrich, St. Louis, MO, USA) and incubated at $37^{\circ} \mathrm{C}$ for $4 \mathrm{~h}$. After removing the supernatant, $150 \mu 1$ DMSO was added and thoroughly mixed. The absorbance was read at $570 \mathrm{~nm}$ using a microplate reader (Sunrise microplate reader; Tecan Group Ltd., Zürich, Switzerland).

Western blot analysis. For mitochondria isolation, the Mitochondria/Cytosol fraction kit (BioVision, Inc., Milpitas, CA, USA) was used according to the manufacturer's instructions. Proteins from the whole SW480 cells, cytosolic fractions and mitochondrial fractions were separated by $12.5 \%$ sodium dodecyl sulfate polyacrylamide gel electrophoresis (SDS-PAGE) and transferred to a PVDF membrane (Millipore, Billerica, MA, USA). Membranes were blocked with 5\% skim milk for $1 \mathrm{~h}$ at room temperature and incubated overnight at $4^{\circ} \mathrm{C}$ with primary antibodies (caspase- 8 , caspase- 9 , caspase- 3 , BID, cytochrome $c$, Smac/DIABLO and $\beta$-actin, all of them were purchased from Cell Signaling Technology, Danvers, MA, USA) at a 1:1,000 dilution. The membranes were subsequently incubated with a horseradish peroxidase (HRP)conjugated secondary antibody (Cell Signaling Technology) at a 1:2,000 dilution for $2 \mathrm{~h}$ at room temperature. The blots were then detected with an enhanced chemiluminescence detection kit (Pierce, Rockford, IL, USA).

Apoptosis assay. For apoptosis analysis, $24 \mathrm{~h}$ post-transfection of RNAs, the cells were reseeded in 6-well plates at a density of $5 \times 10^{5}$ cells/well. Subsequently, after the cells were treated with TRAIL for $48 \mathrm{~h}$, they were stained with FITC-Annexin V and propidium iodide (PI) according to the manufacturer's instructions (Sigma-Aldrich). The percentage of apoptotic cells was quantified using a flow cytometry (Beckman Coulter, Inc., Brea, CA, USA).

Measurement of mitochondrial membrane potential (MMP, $\left.\Delta \Psi_{m}\right)$. For MMP measurement, $24 \mathrm{~h}$ post-transfection of RNAs, the cells were reseeded in 6-well plates at a density of $5 \times 10^{5}$ cells/well. Subsequently, after the cells were treated with TRAIL for $48 \mathrm{~h}$, SW480 cells were incubated with $10 \mu \mathrm{g} / \mathrm{ml}$ JC-1 (Invitrogen) for $10 \mathrm{~min}$ at $37^{\circ} \mathrm{C}$ and then assessed for red fluorescence using a flow cytometry. 

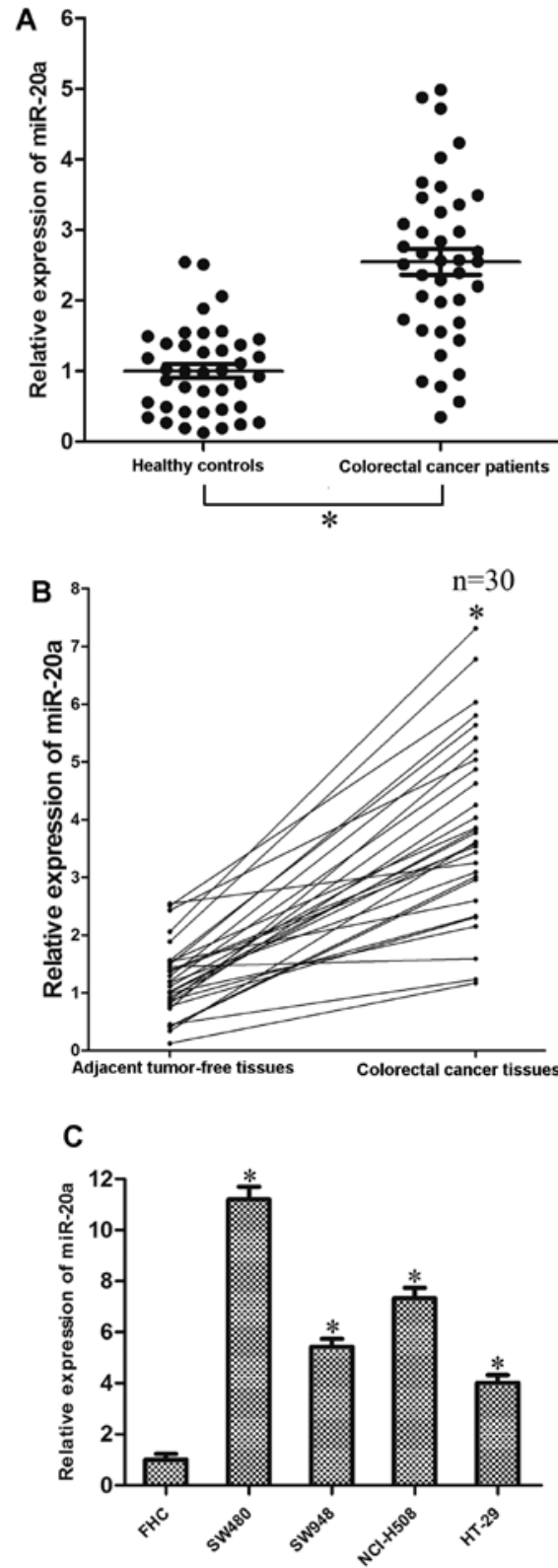

Figure 1. Expression of miR-20a in colorectal cancer. (A) Expression of miR-20a in the serum of colorectal cancer patients and healthy controls was detected by qRT-PCR analysis; ${ }^{*} \mathrm{P}<0.05$. (B) Expression of miR-20a in 30 pairs of colorectal cancer tissues and their adjacent tumor-free tissues was detected by qRT-PCR analysis; "P<0.05. (C) QRT-PCR showed that SW480, SW948, NCI-H508 and HT-29 cells expressed higher levels of miR-20a compared with FHC cells. ${ }^{*} \mathrm{P}<0.05$ vs. FHC cells.

Statistical analysis. All data are expressed as the mean \pm standard deviation and were carried out by three independent experiments. The statistical significance of difference between the groups were determined using the Student's t-test performed by SPSS 13.0 software. $\mathrm{P}<0.05$ was considered statistically significant.

\section{Results}

miR-20a is upregulated in colorectal cancer. To investigate the potential role of miR-20a in colorectal cancer, we analyzed the expression level of miR-20a in colorectal cancer tissues and the adjacent tumor-free tissues, as well as the serum samples of colorectal cancer patients and normal controls. As shown in Fig. 1A, the average expression level of miR-20a was significantly increased in the serum of colorectal cancer patients compared with the healthy controls. Furthermore, among the 30 patients with colorectal cancer, miR-20a was upregulated in tumor tissues compared with the adjacent tumor-free tissues (Fig. 1B). Consistent with the above results, miR-20a expression level was upregulated in all four examined colorectal cancer cell lines (SW480, SW948, NCI-H508 and HT-29) compared with the FHC which is the normal colorectal epithelial cell line (Fig. 1C).

Anti-miR-20a increases the TRAIL sensitivity via caspasedependent apoptosis pathway. To explore the biological roles of miR-20a in TRAIL treatment for colorectal cancer, SW480 cells were transfected with miR-20a mimics or inhibitors. Since the miR-20a mimics or inhibitors significantly changed the level of miR-20a in SW480 cells (Fig. 2A), the combination treatment with these RNAs and TRAIL was performed. As shown in Fig. 2B, knockdown of miR-20a sensitized the SW480 cells to TRAIL-induced cell death, whereas the miR-20a mimics decreased the antitumor effect of TRAIL. These results suggested that miR-20a is associated with the TRAIL sensitivity in colorectal cancer. To study the mechanism by which anti-miR-20a enhanced the TRAIL-induced cell death, we treated the SW480 cells with anti-miR-20a and TRAIL, either alone or in combination, and then the cells were harvested and stained by Annexin V and PI. As shown in Fig. 2C, co-treatment with anti-miR-20a and TRAIL induced apoptosis more significantly compared with the either anti-miR-20a or TRAIL alone treatment group, suggesting the apoptosis pathway was involved in anti-miR-20a associated cell death induced by TRAIL. Notably, anti-miR-20a plus TRAIL significantly activated caspase- 8 , caspase- 9 and caspase-3, whereas TRAIL alone treatment group significantly triggered caspase- 8 besides caspase- 9 and caspase-3 (Fig. 2D). Furthermore, as shown in Fig. 2E, anti-miR-20a plus TRAIL rapidly triggered caspase- 8 in SW480, whereas caspase-9 and caspase- 3 were activated after $12 \mathrm{~h}$, suggesting caspase- 8 acted as the initiator caspase. To investigate this hypothesis, SW480 cells were treated with the caspase-8-specific inhibitor z-IETD-fmk (17) before the combined treatment of antimiR-20a with TRAIL. We found the z-IETD-fmk inhibited the activation of caspase- 8 , as well as caspase- 9 and caspase- 3 , indicating that the caspase- 9 and caspase- 3 were downstream of caspase-8 (Fig. 2F). Taken together, these findings demonstrated that the activation of caspase- 9 and caspase-3, which was initiated by the caspase- 8 activation, was involved in antimiR-20a associated TRAIL-induced apoptosis.

$B I D$ is the target of miR-20a in colorectal cancer. To understand how miR-20a facilitates TRAIL-induced apoptosis, we used TargetScan database to help identify miR-20a targets in SW480. Of these target genes that were predicted by this database, it was revealed that BID is one of the possible target genes of miR-20a (Fig. 3A). BID is a known BH3-only Bcl-2 family member which is the key regulator for linking the extrinsic and mitochondrial (intrinsic) pathway of apoptosis (18). Therefore, we speculated that the gene of BID is a potential target of miR-20a. To investigate this hypothesis, we cloned 

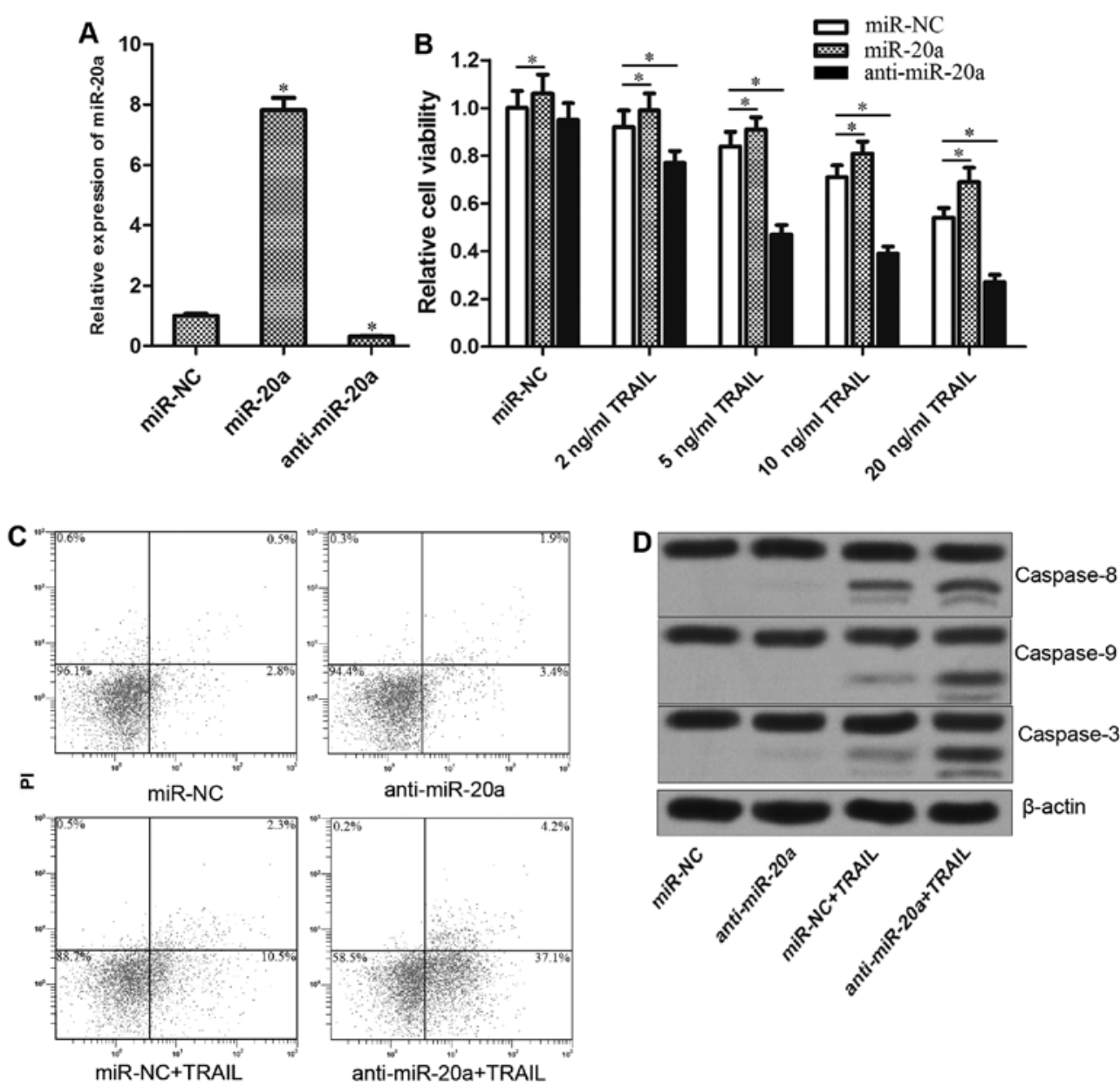

Annexin V
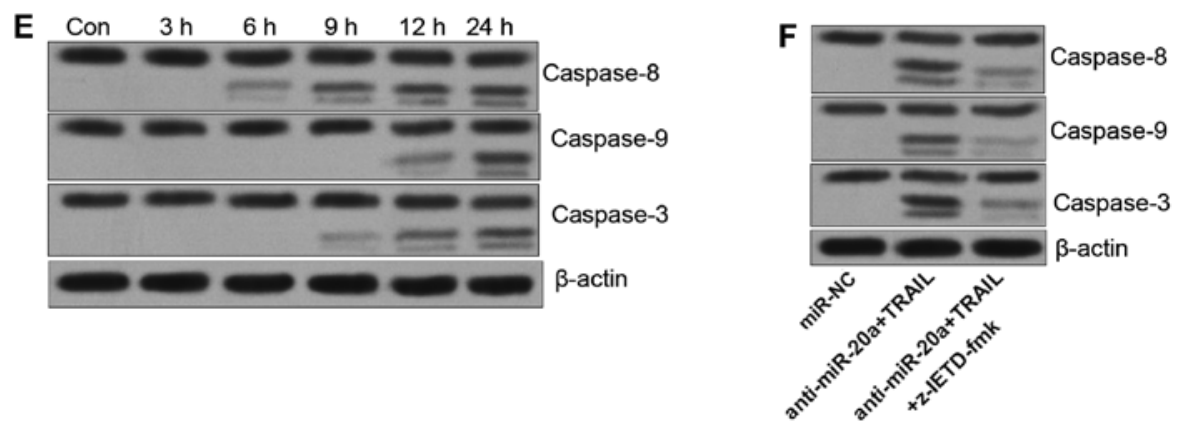

Figure 2. Anti-miR-20a increases the TRAIL sensitivity via caspase-dependent apoptosis pathway. (A) The transfection efficiency of miR-20a mimics or inhibitors was evaluated by RT-qPCR after the SW480 cells were transfected with them for $24 \mathrm{~h}$. (B) SW480 cells were transfected with miR-20a or antimiR-20a. Twenty-four hours after transfection, cells were treated with TRAIL $(5 \mathrm{ng} / \mathrm{ml})$ for $48 \mathrm{~h}$. The relative cell viability was determined by MTT assay. "P<0.05. (C) After the SW480 cells were treated with anti-miR-20a, these cells were stained with Annexin V/PI to measure the ratio of apoptotic cells through FACS. (D) Western blot analysis was performed to detect the activation of caspase- 8 , caspase- 9 and caspase- 3 after the anti-miR-20a and TRAIL (5 ng/ml) treatment. (E) The SW480 cells were collected after they were treated with anti-miR-20a plus TRAIL $(5 \mathrm{ng} / \mathrm{ml})$ for 3, 6, 9, 12 and $24 \mathrm{~h}$. Western blot analysis was then performed to detect the activation of caspase-8, caspase-9 and caspase-3. (F) SW480 cells were treated with anti-miR-20a plus TRAIL (5 ng/ml) in the presence or absence of $20 \mu \mathrm{M}$ z-IETD-fmk which is the caspase-8-specific inhibitor, caspase-8, caspase- 9 and caspase-3 were then detected by western blot analysis.

the BID 3'-UTR sequences containing the predicted target site of miR-20a into a luciferase reporter vector. Luciferase assay revealed that co-transfection of miR-20a mimics and luciferase reporter with wild-type of BID 3'-UTR significantly decreased the luciferase activities, whereas the anti-miR-20a increased the luciferase activities. However, mutation of the putative miR-20a sites in the 3'-UTR of BID abrogated luciferase responsiveness to miR-20a (Fig. 3B). To confirm that
miR-20a regulates the expression of BID, the BID protein was measured after the miR-20a mimics or antagonist was transfected into SW480 cells. As shown in Fig. 3C, miR-20a mimics decreased the expression of BID, whereas the transfection of anti-miR-20a increased the BID protein level in SW480 cells. Taken together, these results suggested that the BID gene is a functional target of miR-20a, which may be associated with the TRAIL sensitivity in colorectal cancer. 
A
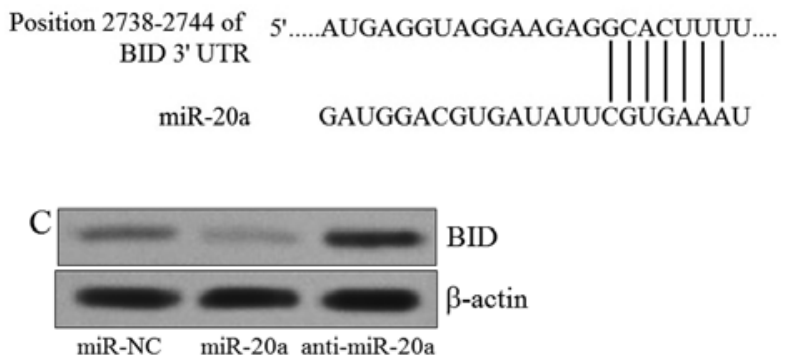

B

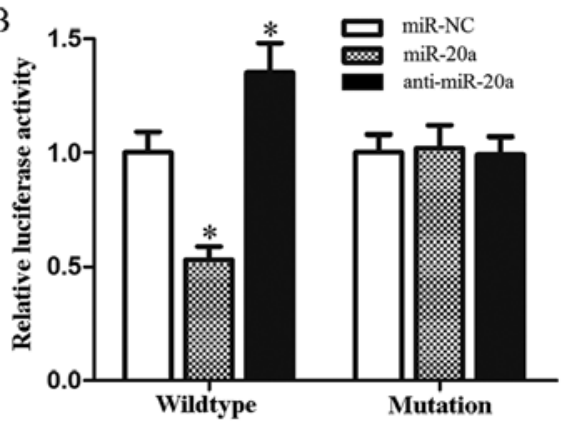

Figure 3. BID is the target of miR-20a in SW480. (A) BID mRNA 3'-untranslated regions containing potential miR-20a binding site is predicted by the TargetScan database. (B) Luciferase assays in SW480 cells. Cells were cotransfected with wild-type/mutant 3'-UTR of BID with miR-20a mimics or antimiR-20a as indicated. Forty-eight hours post-transfection, luciferase activity was detected using Dual-luciferase reporter assay system according to the manufacturer's instruction. ${ }^{*} \mathrm{P}<0.05$ vs. miR-NC group. (C) Western blot analyses showed transfection of miR-20a mimics or anti-miR-20a regulated the expression of BID in SW480 cells.
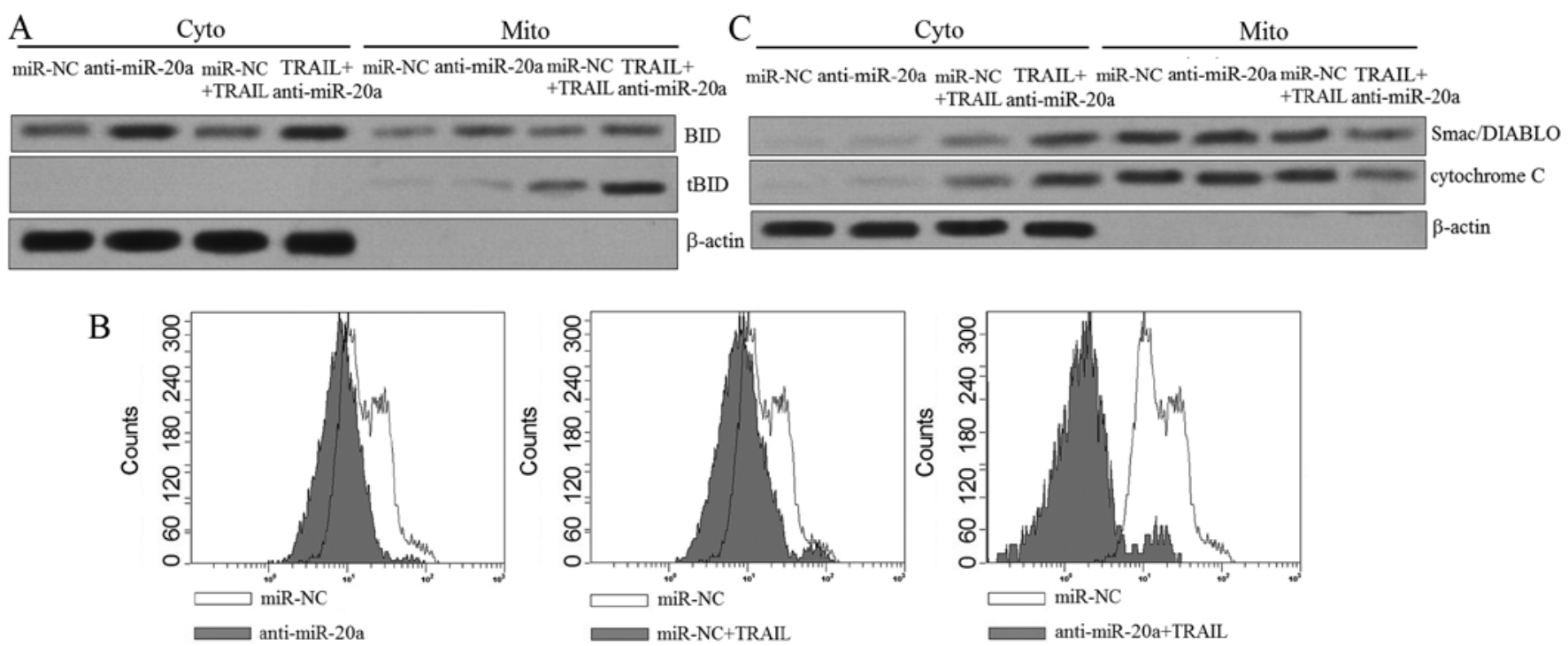

Figure 4. Co-treatment with anti-miR-20a and TRAIL triggers the translocation of tBid and the induction of mitochondrial apoptosis. (A) After separating the mitochondria from the cytoplasm in SW480 cells, western blot assay was performed to detect the protein level of BID or tBID in cytosolic fraction and mitochondrial fraction of SW480 cells. (B) The mitochondrial membrane potential $\left(\Delta \Psi_{\mathrm{m}}\right)$ of SW480 cells treated with anti-miR-20a and TRAIL was detected using JC-1 staining on flow cytometry. (C) Smac/DIABLO and cytochrome $c$ were released from the mitochondria into the cytoplasm due to the combination of TRAIL with anti-miR-20a.

Knockdown of miR-20a increases the translocation of tBid from the cytosolic fraction to the mitochondria. It is acknowledged that BID is the substrate of caspase- 8 activated by TRAIL (19). As we confirmed that BID is the target of miR-20a, we speculated that transfection of anti-miR-20a may expand the TRAIL-induced apoptosis via promoting the translocation of tBid from the cytosolic fraction to the mitochondria. We therefore separated the mitochondria from the cytoplasm in SW480 cells treated with anti-miR-20a and TRAIL, and then the western blot assays were performed. As shown in Fig. 4A, transfection of miR-20a significantly increased the BID expression level in the cytosolic fraction and promoted the translocation of $\mathrm{tBid}$ to the mitochondria in SW480 cells. Since the translocation of $\mathrm{tBid}$ induced mitochondria apoptosis, we measured the mitochondrial membrane potential of SW480 cells by JC-1 staining. As expected, although the anti-miR-20a alone hardly influenced $\Delta \Psi_{\mathrm{m}}$, it significantly facilitated TRAIL to damage the mitochondria of SW480 cells (Fig. 4B), resulting in the release of mitochondria-derived pro-apoptotic inducers such as the cytochrome $c$ and Smac/ DIABLO (Fig. 4C). Taken together, these results suggested the dysfunction of mitochondria is the reason why anti-miR-20a increased the sensitivity of SW480 to TRAIL-induced cell death.

Mitochondrial apoptosis is induced by the combination of TRAIL and anti-miR-20a dependent on the upregulation of BID. Since the activation of caspase- 9 and caspase- 3 is the downstream incidence of mitochondria damage (19), we knoced down BID by its specific siRNA before the SW480 cells were treated with anti-miR-20a plus TRAIL. As we observed, although BID siRNA did not influence the activation of caspase-8, it significantly inhibited the cleavage of caspase-9 and caspase-3 (Fig. 5A). As a result, the apoptotic rate of treated SW480 cells was also decreased by knockdown of BID (Fig. 5B). In summary, these results proved 


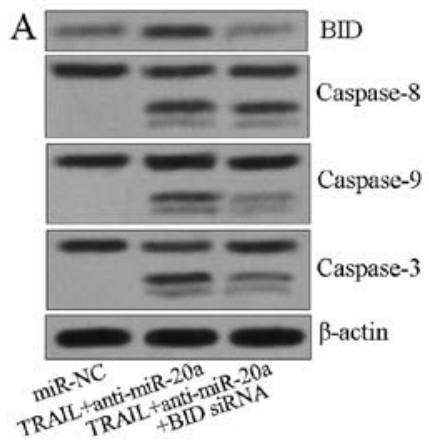

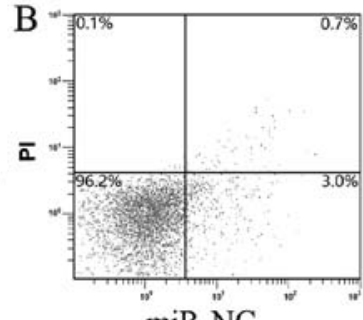

miR-NC

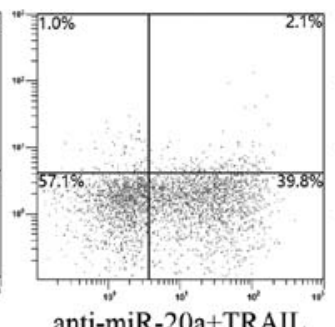

anti-miR-20a+TRAIL

Annexin V

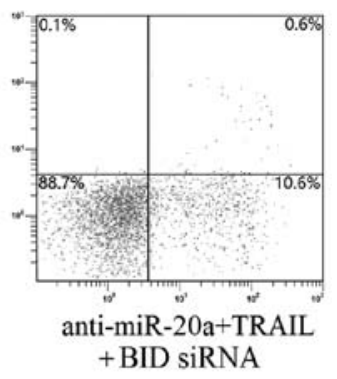

+ BID siRNA

Figure 5. (A) Knockdown of BID by RNA interference impaired the cleavage of caspase- 9 and caspase-3 without influencing the activation of caspase-8 in SW480 cells co-treated with anti-miR-20a and TRAIL. (B) Knockdown of BID decreased the apoptotic rate of SW480 cells co-treated with anti-miR-20a and TRAIL. Cells were analyzed using Annexin V staining on flow cytometry.
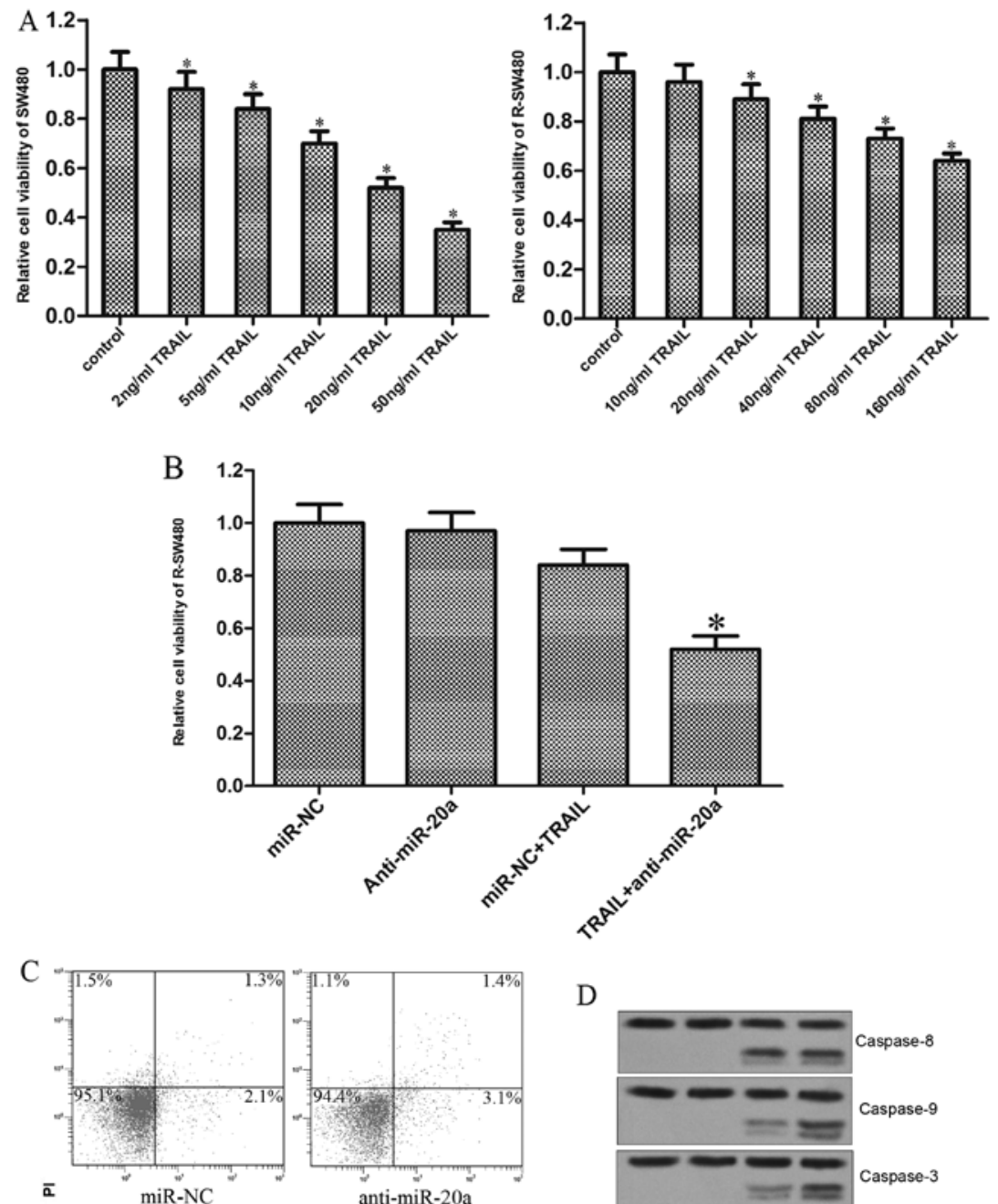

$\mathrm{D}$
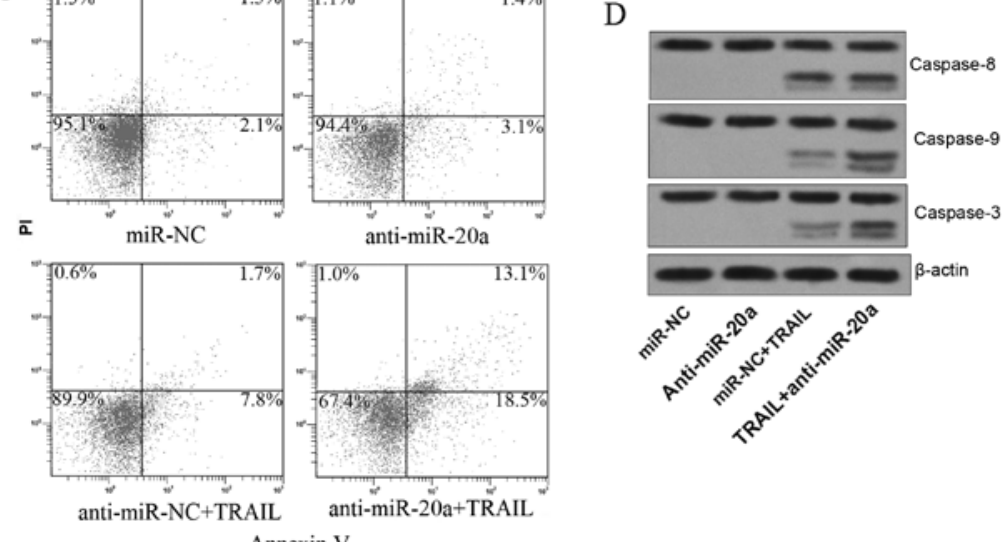

Annexin V

Figure 6. Knockdown of miR-20a reverses the TRAIL resistance in R-SW480. (A) MTT assay was performed to evaluate the sensitivity of SW480 and R-SW480 to TRAIL. (B) Anti-miR-20a significantly increased the sensitivity of R-SW480 cells to TRAIL (40 ng/ml). (C) Knockdown of miR-20a increased the apoptotic rate of R-SW480 cells treated with TRAIL ( $40 \mathrm{ng} / \mathrm{ml}$ ). Apoptosis was analyzed using Annexin V staining on flow cytometry. (D) TRAIL-treated R-SW480 cells showed obvious activation of caspase-9 and caspase-3 due to the knockdown of miR-20a. Western blot analysis was performed to detect the cleavage of caspase-8, caspase-9 and caspase-3. 
the conclusion that knockdown of miR-20a expanded the TRAIL-induced apoptosis via BID/mitochondrial pathway in colorectal cancer.

Knockdown of miR-20a reverses the TRAIL resistance induced by continuous exposure in colorectal cancer. Since the preceding results proved that knockdown of miR-20a increased the sensitivity of SW480 cells to TRAIL, we next investigate whether the anti-miR-20a reversed the TRAIL resistance induced by repeated treatment in colorectal cancer cells. As shown in Fig. 6A, the established TRAIL resistant SW480 cells (R-SW480) showed obvious resistance to TRAIL. Notably, knockdown of miR-20a in R-SW480 significantly resensitized the cells to TRAIL-induced cell death (Fig. 6B). Similarly, although the R-SW480 cells were resistant to TRAIL-induced apoptosis, transfection of antimiR-20a impaired the tolerance, followed by strong apoptosis (Fig. 6C). Furthermore, similarly to the parental SW480, the TRAIL-treated R-SW480 cells also showed obvious activation of caspase-9 and caspase-3 due to the knockdown of miR-20a (Fig. 6D). Taken together, our data strongly suggested that miR-20a is associated with the TRAIL sensitivity in colorectal cancer, knockdown of which can reverse the TRAIL resistance through BID/mitochondrial pathway.

\section{Discussion}

miRNAs are considered as important regulators in substantial cellular processes, including tumorigenesis (20). In addition, previous reports have indicated that miRNAs are related to cancer sensitivity to chemotherapy $(21,22)$. In the present study, we demonstrated that miR-20a was upregulated in colorectal cancer tissues and cell lines. Moreover, knockdown of miR-20a by its specific antagonist was unexpectedly efficient in killing SW480 cells when it was combined with TRAIL. In previous studies, miR-20a was reported to act as an oncomiR in multiple cancers. For instance, overexpression of miR-20a was reported to be associated with the enhancement of the tumor metastasis of colorectal cancer cells (23). In non-small cell lung cancer (NSCLC), the iron exporter ferroportin (FPN), which acts as a tumor suppressor by inhibiting the proliferation and colorectaly formation of cancer cells, was downregulated by the high level of miR-20a (24). Despite the convincing evidence that miR-20a promotes the development of tumors, this study first observed the potential use of miR-20a antagonist as sensitization agent to enhance the antitumor effect of TRAIL in colorectal cancer.

Apoptosis via the TRAIL pathway requires the formation of the death-inducing signaling complex (DISC), which recruits procaspase- 8 and activate it. As the results, the extrinsic apoptosis could be induced directly by the cleaved caspase-8. Importantly, however, the activated caspase- 8 also acts as a bridging element to trigger the mitochondrial pathway of apoptosis, which is also called endogenous apoptosis (25-27). In the present study, we found the combination with miR-20a antagonist and TRAIL induced significant cleavage of caspase-9 and caspase-3, whereas their cleavage was slight in SW480 cells when treated with TRAIL alone. Furthermore, we discovered that the activation of caspase- 9 and caspase- 3 followed caspase- 8 activation. Therefore, we declared that anti-miR-20a could promote TRAIL-induced apoptosis via mitochondrial pathway in colorectal cancer.

BID (BH3 interacting domain death agonist), which is the BH3-only protein in Bcl-2 family, acts as a bridging element between the death receptor signaling and the mitochondrial apoptosis (26). Following the interaction between death receptor and the ligands such as TRAIL, BID is cleaved by caspase- 8 to become the activated form of protein termed truncated BID (tBID) $(28,29)$. After the tBID translocates to the mitochondria, the conformation of Bax and Bak is changed to form the Bax/Bak pores, which lead to the mitochondrial outer membrane permeabilization and mitochondrial depolarization (decrease of $\left.\Delta \Psi_{\mathrm{m}}\right)(30,31)$. As a result, the mitochondriaderived pro-apoptotic inducers (such as the cytochrome $c$ and Smac/DIABLO) are released into the cytosol, followed by the activation of effector caspases (such as caspase- 9 and caspase-3) and apoptosis $(7,32)$.

Previous studies have suggested that the activation of BID is an important strategy in cancer therapy $(33,34)$. In the present study, we also demonstrated that the miR-20a antagonist promoted the TRAIL-dependent mitochondrial apoptosis by regulating the expression of BID directly. As a result of BID upregulation, the miR-20a antagonist promoted the translocation of tBID in TRAIL-treated SW480 cells, followed by the mitochondrial depolarization and the release of cytochrome $c$ and Smac/DIABLO. Previous studies indicated that the cytochrome $c$ in cytosol triggers caspase-3 activation by inducing the formation of Apaf-1/caspase-9 apoptosome, the Smac/ DIABLO in cytosol neutralizes the inhibitor of apoptosis proteins (IAPs) (35), the mitochondrial apoptosis occurred finally because of the combination of anti-miR-20a with TRAIL.

Drug-resistance is a major challenge to effective TRAIL treatment (36). Therefore, we finally tested the role of miR-20a antagonist in reversing the TRAIL resistance in colorectal cancer. We observed that the anti-miR-20a can increase the sensitivity of experimental TRAIL-resistant SW480 to TRAIL-induced cell death, suggesting the attractive prospect of miR-20a antagonist on colorectal cancer treatment with TRAIL.

In summary, we have provided several pieces of evidence suggesting that miR-20a is associated with the TRAIL sensitivity in colorectal cancer by regulating the pro-apoptotic gene of BID. The identification of miR-20a antagonist as the promoter of TRAIL might have significant therapeutic potential and may provide a novel strategy for overcoming drug-resistance.

\section{Acknowledgements}

The present study was supported by the Wenzhou Science and Technology Bureau (grant no. Y20150050).

\section{References}

1. Siegel RL, Miller KD and Jemal A: Cancer statistics, 2015. CA Cancer J Clin 65: 5-29, 2015.

2. Manfredi S, Lepage C, Hatem C, Coatmeur O, Faivre J and Bouvier AM: Epidemiology and management of liver metastases from colorectal cancer. Ann Surg 244: 254-259, 2006. 
3. Bao S, Ouyang G, Bai X, Huang Z, Ma C, Liu M, Shao R, Anderson RM, Rich JN and Wang XF: Periostin potently promotes metastatic growth of colon cancer by augmenting cell survival via the Akt/PKB pathway. Cancer Cell 5: 329-339, 2004.

4. Yi HJ, Hong KS, Moon N, Chung SS, Lee RA and Kim KH: Acute hyperammonemic encephalopathy after 5-fluorouracil based chemotherapy. Ann Surg Treat Res 19: 264-271, 2006.

5. Fuchs D, Metzig M, Bickeböller M, Brandel C and Roth W: The G $\beta 5$ protein regulates sensitivity to TRAIL-induced cell death in colon carcinoma. Oncogene 34: 2753-2763, 2015.

6. Hwang JS, Lee HC, Oh SC, Lee DH and Kwon KH: Shogaol overcomes TRAIL resistance in colon cancer cells via inhibiting of survivin. Tumour Biol 36: 8819-8829, 2015.

7. Laussmann MA,Passante E, Hellwig CT, Tomiczek B,Flanagan L, Prehn JH, Huber HJ and Rehm M: Proteasome inhibition can impair caspase- 8 activation upon submaximal stimulation of apoptotic tumor necrosis factor-related apoptosis inducing ligand (TRAIL) signaling. J Biol Chem 287: 14402-14411, 2012.

8. Walczak H, Miller RE, Ariail K, Gliniak B, Griffith TS, Kubin M, Chin W, Jones J, Woodward A, Le T, et al: Tumoricidal activity of tumor necrosis factor-related apoptosis-inducing ligand in vivo. Nat Med 5: 157-163, 1999.

9. Thorburn A, Behbakht K and Ford H: TRAIL receptor-targeted therapeutics: Resistance mechanisms and strategies to avoid them. Drug Resist Updat 11: 17-24, 2008.

10. Finlay D, Vamos M, González-López M, Ardecky RJ, Ganji SR, Yuan H, Su Y, Cooley TR, Hauser CT, Welsh K, et al: Small-molecule IAP antagonists sensitize cancer cells to TRAIL-induced apoptosis: Roles of XIAP and cIAPs. Mol Cancer Ther 13: 5-15, 2014.

11. Lee RC, Feinbaum RL and Ambros V: The C. elegans heterochronic gene lin-4 encodes small RNAs with antisense complementarity to lin-14. Cell 75: 843-854, 1993.

12. Bartel DP: MicroRNAs: Target recognition and regulatory functions. Cell 136: 215-233, 2009.

13. He H, Tian W, Chen $\mathrm{H}$ and Jiang K: MiR-944 functions as a novel oncogene and regulates the chemoresistance in breast cancer. Tumour Biol 37: 1599-1607, 2016.

14. Zheng Y, Lv X, Wang X, Wang B, Shao X, Huang Y, Shi L, Chen Z, Huang J and Huang P: MiR-181b promotes chemoresistance in breast cancer by regulating Bim expression. Oncol Rep 35: 683-690, 2016.

15. Kim AD, Zhang R, Han X, Kang KA, Piao MJ, Maeng YH, Chang WY and Hyun JW: Involvement of glutathione and glutathione metabolizing enzymes in human colorectal cancer cell lines and tissues. Mol Med Rep 12: 4314-4319, 2015.

16. Livak KJ and Schmittgen TD: Analysis of relative gene expression data using real-time quantitative PCR and the 2(-Delta Delta C(T)) method. Methods 25: 402-408, 2001.

17. Yeh CC, Deng YT, Sha DY,Hsiao M and Kuo MY: Suberoylanilide hydroxamic acid sensitizes human oral cancer cells to TRAILinduced apoptosis through increase DR5 expression. Mol Cancer Ther 8: 2718-2725, 2009 .

18. Yin XM: Bid, a BH3-only multi-functional molecule, is at the cross road of life and death. Gene 369: 7-19, 2006.

19. Kantari $\mathrm{C}$ and Walczak H: Caspase- 8 and bid: Caught in the act between death receptors and mitochondria. Biochim Biophys Acta 1813: 558-563, 2011.
20. Frixa T, Donzelli S and Blandino G: Oncogenic MicroRNAs: Key players in malignant transformation. Cancers (Basel) 7 2466-2485, 2015.

21. Ye Z, Hao R, Cai Y, Wang X and Huang G: Knockdown of miR-221 promotes the cisplatin-inducing apoptosis by targeting the BIM-Bax/Bak axis in breast cancer. Tumour Biol 37: 4509-4515, 2016.

22. Xie X, Hu Y, Xu L, Fu Y, Tu J, Zhao H, Zhang S, Hong R and $\mathrm{Gu} \mathrm{X}$ : The role of miR-125b-mitochondria-caspase-3 pathway in doxorubicin resistance and therapy in human breast cancer. Tumour Biol 36: 7185-7194, 2015.

23. Xu T, Jing C, Shi Y, Miao R, Peng L, Kong S, Ma Y and Li L: microRNA-20a enhances the epithelial-to-mesenchymal transition of colorectal cancer cells by modulating matrix metalloproteinases. Exp Ther Med 10: 683-688, 2015.

24. Babu KR and Muckenthaler MU: miR-20a regulates expression of the iron exporter ferroportin in lung cancer. J Mol Med (Berl) 94: 347-359, 2016.

25. Salvesen GS and Walsh CM: Functions of caspase 8: The identified and the mysterious. Semin Immunol 26: 246-252, 2014.

26. Li H, Zhu H, Xu CJ and Yuan J: Cleavage of BID by caspase 8 mediates the mitochondrial damage in the Fas pathway of apoptosis. Cell 94: 491-501, 1998.

27. Fulda $\mathrm{S}$ and Debatin KM: Extrinsic versus intrinsic apoptosis pathways in anticancer chemotherapy. Oncogene 25: 4798-4811, 2006.

28. Billen LP, Shamas-Din A and Andrews DW: Bid: A Bax-like BH3 protein. Oncogene 27 (Suppl 1): S93-S104, 2008.

29. Orzechowska EJ, Girstun A, Staron K and TrzcinskaDanielewicz J: Synergy of BID with doxorubicin in the killing of cancer cells. Oncol Rep 33: 2143-2150, 2015.

30. Eskes R, Desagher S, Antonsson B and Martinou JC: Bid induces the oligomerization and insertion of Bax into the outer mitochondrial membrane. Mol Cell Biol 20: 929-935, 2000.

31. Wei MC, Lindsten T, Mootha VK, Weiler S, Gross A, Ashiya M, Thompson CB and Korsmeyer SJ: tBID, a membrane-targeted death ligand, oligomerizes BAK to release cytochrome c. Genes Dev 14: 2060-2071, 2000.

32. Billard C: Design of novel BH3 mimetics for the treatment of chronic lymphocytic leukemia. Leukemia 26: 2032-2038, 2012.

33. Boonyarat $\mathrm{C}$, Yenjai $\mathrm{C}$, Vajragupta $\mathrm{O}$ and Waiwut $\mathrm{P}$ : Heptaphylline induces apoptosis in human colon adenocarcinoma cells through bid and Akt/NF-кB (p65) pathways. Asian Pac J Cancer Prev 15: 10483-10487, 2014.

34. Wu CS, Chen GS, Lin PY, Pan IH, Wang ST, Lin SH, Yu HS and Lin CC: Tazarotene induces apoptosis in human basal cell carcinoma via activation of caspase-8/t-Bid and the reactive oxygen species-dependent mitochondrial pathway. DNA Cell Biol 33: 652-666, 2014.

35. Saelens X, Festjens N, Vande Walle L, van Gurp M, van Loo G and Vandenabeele P: Toxic proteins released from mitochondria in cell death. Oncogene 23: 2861-2874, 2004.

36. Trivedi R and Mishra DP: Trailing TRAIL resistance: Novel targets for TRAIL sensitization in cancer cells. Front Oncol 5: $69,2015$. 